\title{
Some Switching Invariant Prime Graphs
}

\author{
Samir K. Vaidya ${ }^{1}$, Udayan M. Prajapati ${ }^{2}$ \\ ${ }^{1}$ Department of Mathematics, Saurashtra University, Rajkot, India \\ ${ }^{2}$ St. Xavier's College, Ahmedabad, India \\ Email: samirkvaidya@yahoo.co.in, udayan64@yahoo.com
}

Received November 12, 2011; revised December 10, 2011; accepted December 31, 2011

\begin{abstract}
We investigate prime labeling for some graphs resulted from switching of a vertex. We discuss switching invariance of some prime graphs and prove that the graphs obtained by switching of a vertex in $P_{n}$ and $K_{1, n}$ admit prime labeling. Moreover we discuss prime labeling for the graph obtained by switching of vertex in wheel $W_{n}$.
\end{abstract}

Keywords: Prime Labeling; Switching of a Vertex; Switching Invariance

\section{Introduction and Definitions}

We begin with simple, finite, undirected and non-trivial graph $G=(V(G), E(G))$, with vertex set $V(G)$ and edge set $E(G)$. Throughout this work $C_{n}$ denotes the cycle with $n$ vertices and $P_{n}$ denotes the path of $n$ vertices. In wheel $W_{n}=C_{n}+K_{1}$ the vertex corresponding to $K_{1}$ is called apex vertex and the vertices corresponding to $C_{n}$ are called rim vertices where $n \geq 3$. The star $K_{1, n}$ is a graph with one vertex of degree $n$ called apex and $n$ vertices of degree one (pendant vertices). Throughout this paper $|V(G)|$ and $|E(G)|$ are the cardinality of vertex set and edge set respectively.

For various graph theoretic notations and terminology we follow Gross and Yellen [1] while for number theory we follow Niven and Zuckerman [2]. We will give brief summary of definitions and other information which are useful for the present investigations.

Definition 1.1: If the vertices of the graph are assigned values subject to certain conditions then it is known as graph labeling.

Vast amount of literature is available in printed as well as in electronic form on different types of graph labeling. More than 1300 research papers have been published so far in last four decades. For a dynamic survey of graph labeling problems along with extensive bibliography we refer to Gallian [3].

Definition 1.2: A prime labeling of a graph $G$ is an injective function $f: V(G) \rightarrow\{1,2,3, \cdots,|V(G)|\}$ such that for every pair of adjacent vertices $u$ and $v$, $\operatorname{gcd}(f(u), f(v))=1$. The graph which admits a prime labeling is called a prime graph.

The notion of a prime labeling was originated by
Entringer and was discussed in a paper by Tout et al. [4]. Many researchers have studied prime graphs. For e.g. Fu and Huang [5] have proved that $P_{n}$ and $K_{1, n}$ are prime graphs. Lee et al [6] have proved that $W_{n}$ is a prime graph if and only if $n$ is even. Deretsky et al. [7] have proved that $C_{n}$ is a prime graph.

Definition 1.3: A vertex switching $G_{v}$ of a graph $G$ is the graph obtained by taking a vertex $v$ of $G$, removing all the edges incident to $v$ and adding edges joining $v$ to every other vertex which are not adjacent to $v$ in $G$.

Definition 1.4: A prime graph $G$ is said to be switching invariant if for every vertex $v$ of $G$, the graph $G_{v}$ obtained by switching the vertex $v$ in $G$ is also a prime graph.

Vaidya and Kanani [8] have established the switching invariance of $C_{n}$ corresponding to prime labeling while in the present paper we investigate further results on prime graphs.

Bertarnad's Postulate 1.5: For every positive integer $n>1$ there is a prime $p$ such that $n<p<2 n$.

\section{Some Results on Prime Labeling with Respect to Vertex Switching Operation}

Observation 2.1: Every prime graph $G$ of order $n$ has at least one vertex $v$ (corresponding to label 1) such that $G_{v}$ is a prime graph.

Observation 2.2: Let $G$ be a prime graph of order $n \geq 2$ with a prime labeling. If $v$ is the vertex corresponding to the largest prime less then or equal to $n$ then $G_{v}$ is a prime graph.

Observation 2.3: Let $G$ be a prime graph of order $n \geq 3$ with a prime labeling and $v$ is any arbitrary 
vertex having prime label from the set $\left\{\left\lfloor\frac{n}{2}\right\rfloor,\left\lfloor\frac{n}{2}\right\rfloor+1, \cdots, n\right\}$ then $G_{v}$ is a prime graph.

Theorem 2.4: $P_{n}$ is switching invariant.

Proof: Let $v_{1}, v_{2}, \cdots, v_{n}$ be consecutive vertices of $P_{n}$. Let $G_{v}$ be the graph obtained by switching a vertex $v$ of $P_{n}$.

For the vertex $v$ we have the following possibilities:

1) $v=v_{1}$ then in $G_{v_{1}}, v_{1}$ is adjacent to

$v_{3}, v_{4}, \cdots, v_{n}$.

Define $f: V\left(G_{v_{1}}\right) \rightarrow\left\{1,2,3, \cdots,\left|V\left(G_{v_{1}}\right)\right|\right\}$ as follows:

$f\left(v_{i}\right)=i$, where $i=1,2, \cdots, n$

Then clearly $f$ is an injection.

For an arbitrary edge $e=a b$ of $G_{v_{1}}$ we claim that $\operatorname{gcd}(f(a), f(b))=1$. Because

a) if $e=v_{1} v_{i}$ for some $i \in\{3,4, \cdots, n\}$ then

$\operatorname{gcd}\left(f\left(v_{1}\right), f\left(v_{i}\right)\right)=\operatorname{gcd}(1, i)=1$;

b) if $e=v_{i} v_{i+1}$ for some $i \in\{2,3, \cdots, n-1\}$ then

$\operatorname{gcd}\left(f\left(v_{i}\right), f\left(v_{i+1}\right)\right)=\operatorname{gcd}(i, i+1)=1$ as $i$ and $i+1$ are consecutive positive integers.

If $v=v_{n}$ the proof is similar as discussed above.

2) $v=v_{i}$ for some $i \in\{2,3, \cdots, n-1\}$. Then in $G_{v_{i}}$, $v_{i}$ is adjacent to all the vertices except $v_{i-1}$ and $v_{i+1}$.

Define $f: V\left(G_{v_{i}}\right) \rightarrow\left\{1,2,3, \cdots,\left|V\left(G_{v_{i}}\right)\right|\right\}$ as follows:

$$
f\left(v_{j}\right)=\left\{\begin{array}{cc}
j+1 & \text { if } j=1,2, \cdots, i-1 ; \\
j & \text { if } j=i+1, i+2, \cdots, n ; \\
1 & \text { if } j=i .
\end{array}\right.
$$

Then clearly $f$ is an injection.

For an arbitrary edge $e=a b$ of $G_{v_{i}}$ we claim that $\operatorname{gcd}(f(a), f(b))=1$.

To prove our claim the following cases are to be considered.

a) If $e=v_{i} v_{j}$ for some $j \in\{1,2, \cdots, i-2\}$ then $\operatorname{gcd}\left(f\left(v_{i}\right), f\left(v_{j}\right)\right)=\operatorname{gcd}(1, j+1)=1$;

b) If $e=v_{i} v_{j}$ for some $j \in\{i+2,3, \cdots, n\}$ then $\operatorname{gcd}\left(f\left(v_{i}\right), f\left(v_{j}\right)\right)=\operatorname{gcd}(1, j)=1$.

c) If $e=v_{j} v_{j+1}$ for some $j \in\{1,2, \cdots, i-2\}$ then $\operatorname{gcd}\left(f\left(v_{j}\right), f\left(v_{j+1}\right)\right)=\operatorname{gcd}(j+1, j+2)=1$ as $j$ and $j+1$ are consecutive positive integers;

d) If $e=v_{j} v_{j+1}$ for some $j \in\{i+2, i+3, \cdots w, n\}$ then $\operatorname{gcd}\left(f\left(v_{j}\right), f\left(v_{j+1}\right)\right)=\operatorname{gcd}(j, j+1)=1$;

Thus in each of the possibilities the graph $G_{v}$ under consideration admits a prime labeling. i.e. $G_{v}$ is a prime graph.

Thus $P_{n}$ and the graph obtained by switching of any vertex in $P_{n}$ are prime graphs. Hence the result.

Illustration 2.5: The prime labeling of the graph obtained by switching a pendant vertex of $P_{5}$ is shown in the Figure 1.

Illustration 2.6: The prime labeling of the graph obtained by switching a vertex of $P_{5}$ which is not a pendant vertex is shown in the Figure 2.

Theorem 2.7: $K_{1, n}$ is switching invariant.

Proof: We will separate two cases:

1) Switching of the apex vertex.

2) Switching of any pendant vertex.

Case 1: If $v$ is the apex vertex of $K_{1, n}$ and $G_{v}$ is the graph obtained by switching the apex vertex $v$ then $G_{v}$ is the null graph $N_{n}$ on $n$ vertices, which does not have any edge. Then obviously it is a prime graph.

Case 2: Let $v_{0}$ be the apex vertex and $v_{1}, v_{2}, \cdots, v_{n}$ be the consecutive pendant vertices of $K_{1, n}$. Let $G_{v_{n}}$ be the graph obtained by switching the pendant vertex $v_{n}$ of $K_{1, n}$. So in $G_{v_{n}}$ every vertex $v_{i}$ other than $v_{0}$ and $v_{n}$ is adjacent to $v_{0}$ and $v_{n}$ only. By Bertrand's postulate of number theory there exists at least one prime $p$ such that $\frac{n}{2} \leq p \leq n+1$ then it is possible to define $f:\left\{v_{0}, v_{1}, v_{2}, \cdots, v_{n}\right\} \rightarrow\{1,2,3, \cdots, n+1\}$ as follows:

$$
f\left(v_{j}\right)=\left\{\begin{array}{cc}
1 & \text { if } j=0 \\
j+1 & \text { if } j=1,2, \cdots p-2 \\
j+2 & \text { if } j=p-1, p, p+1, \cdots, n-1 ; \\
p & \text { if } j=n .
\end{array}\right.
$$

In view of the pattern defined above $f$ admits a prime labeling on $G_{v_{n}}$. Hence $G_{v_{n}}$ is a prime graph.

Thus $K_{1, n}$ and the graph obtained by switching any vertex of $K_{1, n}$ are prime graphs. Hence the result.

Illustration 2.8: The prime labeling of the graph obtained by switching a pendant vertex of $K_{1,6}$ is shown in the Figure 3.

Theorem 2.9: Switching the apex vertex in $W_{n}$ is a prime graph.

Proof: Let $v_{1}, v_{2}, \cdots, v_{n}$ be consecutive rim vertices of $W_{n}$ and $v_{0}$ be the apex vertex of $W_{n}$. Let $G$ be the graph obtained by switching the vertex $v_{0}$. Thus $G$

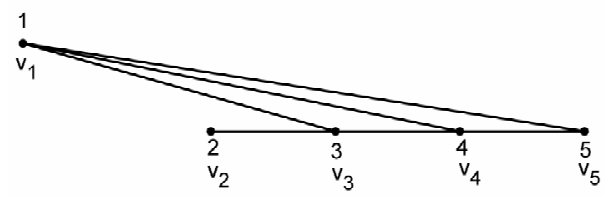

Figure 1. The prime labeling of the graph obtained by switching a pendant vertex of $\boldsymbol{P}_{5}$.

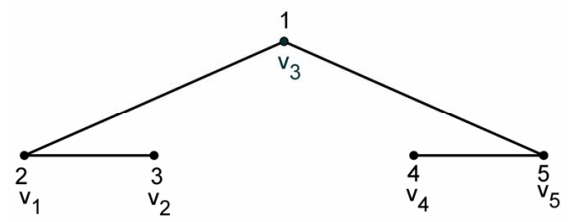

Figure 2. The prime labeling of the graph obtained by switching a vertex of $P_{5}$ which is not a pendant vertex. 


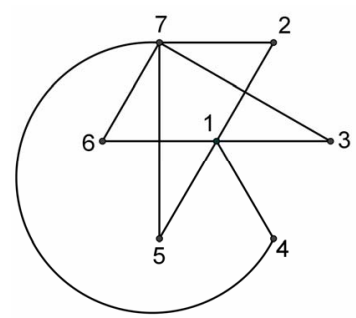

Figure 3. The prime labeling of the graph obtained by switching a pendant vertex of $K_{1,6}$.

is the disjoint union of $C_{n}$ and $K_{1}$.

Define $f: V(G) \rightarrow\{1,2,3, \cdots,|V(G)|\}$ as follows:

$$
f\left(v_{i}\right)=\left\{\begin{array}{cc}
n+1 & \text { if } i=0 ; \\
i & \text { if } i=1,2, \cdots n .
\end{array}\right.
$$

Then clearly $f$ is an injection.

For an arbitrary edge $e=a b$ of $G$ we claim that $\operatorname{gcd}(f(a), f(b))=1$.

1) If $e=v_{i} v_{i+1}$ for some $i \in\{1,2,3, \cdots, n\}$ then

$\operatorname{gcd}\left(f\left(v_{i}\right), f\left(v_{i+1}\right)\right)=\operatorname{gcd}(i, i+1)=1$ as $i$ and $i+1$ are consecutive positive integers.

2) If $e=v_{n} v_{1}$ then $\operatorname{gcd}\left(f\left(v_{n}\right), f\left(v_{0}\right)\right)=\operatorname{gcd}(n, 1)=1$.

Thus in each of the possibilities the graph $G$ admits a prime labeling. i.e. $G$ is a prime graph.

Illustration 2.10: The prime labeling of the graph obtained by switching the apex vertex of $W_{6}$ is shown in the Figure 4.

Theorem 2.11: Switching of a rim vertex of $W_{n}$ is a prime graph if $n+1$ is a prime number.

Proof: Let $v_{1}, v_{2}, \cdots, v_{n}$ be consecutive rim vertices of $W_{n}$ and $v_{0}$ be the apex vertex of $W_{n}$. Let $G$ be the graph obtained by switching the vertex $v_{n}$.

Define $f: V(G) \rightarrow\{1,2,3, \cdots,|V(G)|\}$ as follows:

$f\left(v_{i}\right)=i+1, \forall i=1,2, \cdots, n$ and $f\left(v_{0}\right)=1$.

Then clearly $f$ is an injection.

For an arbitrary edge $e=a b$ of $G$ we claim that $\operatorname{gcd}(f(a), f(b))=1$.

1) If $e=v_{i} v_{i+1}$ for some $i \in\{1,2,3, \cdots, n-2\}$ then $\operatorname{gcd}\left(f\left(v_{i}\right), f\left(v_{i+1}\right)\right)=\operatorname{gcd}(i+1, i+2)=1$ as $i+1$ and $i+2$ are consecutive positive integers.

2) If $e=v_{0} v_{i}$ for some $i \in\{1,2,3, \cdots, n-1\}$ then $\operatorname{gcd}\left(f\left(v_{0}\right), f\left(v_{i}\right)\right)=\operatorname{gcd}(1, i+1)=1$.

3) If $e=v_{n} v_{i}$ for some $i \in\{2,3, \cdots, n-2\}$ then $\operatorname{gcd}\left(f\left(v_{n}\right), f\left(v_{i}\right)\right)=\operatorname{gcd}(n+1, i+1)=1$ as $i+1$ is a positive integer less than the prime number $n+1$.

Thus in each of the possibilities the graph $G$ under consideration admits a prime labeling. i.e. $G$ is a prime graph.

Illustration 2.12: The prime labeling of the graph obtained by switching a rim vertex of $W_{6}$ is shown in the Figure 5.

Proposition 2.13: The graph obtained by switching of

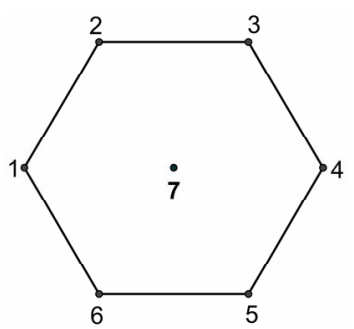

Figure 4. The prime labeling of the graph obtained by switching the apex vertex of $W_{6}$.

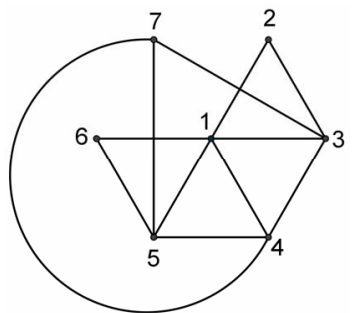

Figure 5. The prime labeling of the graph obtained by switching a rim vertex of $W_{6}$.

a rim vertex in $W_{7}$ is not a prime graph.

Proof: Let $v_{1}, v_{2}, \cdots, v_{7}$ be consecutive rim vertices of $W_{7}$ and $v_{0}$ be the apex vertex of $W_{7}$. Let $G$ be the graph obtained by switching the vertex $v_{7}$.

If possible let $f: V(G) \rightarrow\{1,2,3, \cdots, 8\}$ be a prime labeling. As $v_{7}$ is adjacent to four vertices $v_{2}, v_{3}, v_{4}, v_{5}$ and $v_{0}$ is adjacent to six vertices $v_{1}, v_{2}, v_{3}, v_{4}, v_{5}, v_{6}$, the labels of $v_{7}$ and $v_{0}$ can not be even. Moreover we have to distribute four even labels among six vertices. Therefore at least two adjacent vertices from

$v_{1}, v_{2}, v_{3}, v_{4}, v_{5}, v_{6}$ will receive the even labels which contradicts the fact that $f$ is a prime labeling.

We noticed that it is not easy to discuss the prime labeling of a graph obtained by switching any rim vertex of $W_{n}$ when $n+1$ is a composite number. However we prove a following result and pose a conjecture.

Theorem 2.14: Switching of a rim vertex in $W_{n}$ is a not a prime graph if $n+1$ is an even integer greater than 9.

Proof: Let $v_{1}, v_{2}, \cdots, v_{n}$ be consecutive rim vertices and $v_{0}$ be the apex vertex of $W_{n}$. Let $G$ be the graph obtained by switching the vertex $v_{n}$. If possible assume that there exists a prime labeling

$f: V(G) \rightarrow\{1,2, \cdots, n+1\}$ on $G$.

Observe that in $\{1,2, \cdots, n+1\}$ the number of even integers is equal to the number of odd integers in

$\{1,2, \cdots, n+1\}$ which is $\frac{n+1}{2}$.

If a vertex $v$ is adjacent to at least $n-3$ vertices then it cannot be labeled with even integer otherwise each of its $n-3$ neighbours should receive the labels 
with odd integers. Consequently there should be at least $n-3$ odd integers in $\{1,2, \cdots, n+1\}$ and at the most $n+1-(n-3)=4$ even integers. However, there are at least 5 even integers in $\{1,2,3, \cdots, n\}$, a contradiction.

Consequently each of the vertices $v_{0}$ and $v_{n}$ have at least $n-3$ neighbours must be labeled with an odd integer. Therefore the remaining vertices $v_{1}, v_{2}, \cdots, v_{n-1}$ forms a path $P_{n-1}$ in $G$ and these vertices will receive $\frac{n+1}{2}-2=\frac{n-3}{2}$ odd labels and $\frac{n+1}{2}$ even labels. If $L$ and $M$ denote the number of vertices with even labels and number of vertices with odd labels respectively in $P_{n-1}$ then $L-M=\frac{n+1}{2}-\frac{n-3}{2}=2$. Hence there must be two adjacent vertices in $P_{n-1}$ which will receive even labels which contradicts our assumption that $f$ is a prime labeling of $G$.

Conjecture 2.15: The graph obtained by switching of a rim vertex in $W_{n}$ is a not a prime graph if $n+1$ is a composite odd integer greater than 9.

\section{Concluding Remarks}

The study of prime numbers is of great importance as prime numbers are scattered and there are arbitrarily large gaps in the sequence of prime numbers. If these characteristics are studied in the frame work of graph theory then it is more challenging and exciting as well.

Here we investigate several results on prime labeling of graphs and pose a conjecture. This discussion becomes more relevant as it is carried out in the context of a graph operation namely switching of a vertex. We have studied switching invariant behaviour of some standard graphs.

\section{REFERENCES}

[1] J. Gross and J. Yellen, "Graph Theory and Its Applications,” CRC Press, Boca Raton, 1999.

[2] I. Niven and H. S. Zuckerman, "An Introduction to the Theory of Numbers,” 3rd Edition, Wiley Eastern Limited, New York, 1972.

[3] J. A. Gallian, "A Dynamic Survey of Graph Labeling," The Electronic Journal of Combinatorics, Vol. 17, 2010, \#DS6. http:Ilwww.combinatorics.org $\backslash$ Surveys \ds6.pdf

[4] A. Tout, A. N. Dabboucy and K. Howalla, "Prime Labeling of Graphs,” National Academy Science Letters, Vol. 11, 1982, pp. 365-368.

[5] H. L. Fu and K. C. Huang, “On Prime Labellings,” Discrete Mathematics, Vol. 127, No. 1-3, 1994, pp. 181-186. doi:10.1016/0012-365X(92)00477-9

[6] S. M. Lee, I. Wui and J. Yeh, "On the Amalgamation of Prime Graphs,” Bulletin of the Malaysian Mathematical Science Society (Second Series), Vol. 11, 1988, pp. 59-67.

[7] T. Deretsky, S. M. Lee and J. Mitchem, "On Vertex Prime Labelings of Graphs," Proceedings of the 6th International Conference Theory and Applications of Graphs, Wiley, New York, 1991, pp. 359-369.

[8] S. K. Vaidya and K. K. Kanani, "Prime Labeling for Some Cycle Related Graphs," Journal of Mathematics Rsearch, Vol. 2, No. 2, 2010, pp. 98-103. http:\\ccsenet.org\journal lindex.php\jmrlarticlelview $\backslash 4423$ 14743 